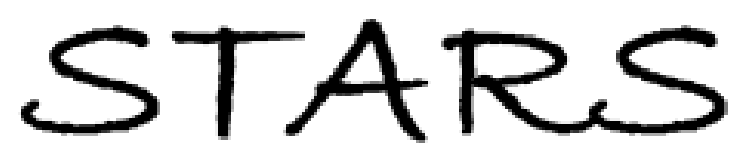

University of Central Florida

STARS

Faculty Bibliography 2010s

Faculty Bibliography

$1-1-2015$

\title{
Imaging of Ankle Replacements
}

Gary M. LiMarzi

Dzmitry M. Fursevich

J. Dean Cole

Christopher W. Wasyliw

Laura W. Bancroft

University of Central Florida

Find similar works at: https://stars.library.ucf.edu/facultybib2010

University of Central Florida Libraries http://library.ucf.edu

This Article is brought to you for free and open access by the Faculty Bibliography at STARS. It has been accepted for inclusion in Faculty Bibliography 2010 s by an authorized administrator of STARS. For more information, please contactSTARS@ucf.edu.

\section{Recommended Citation}

LiMarzi, Gary M.; Fursevich, Dzmitry M.; Cole, J. Dean; Wasyliw, Christopher W.; and Bancroft, Laura W., "Imaging of Ankle Replacements" (2015). Faculty Bibliography 2010s. 6664.

https://stars.library.ucf.edu/facultybib2010/6664

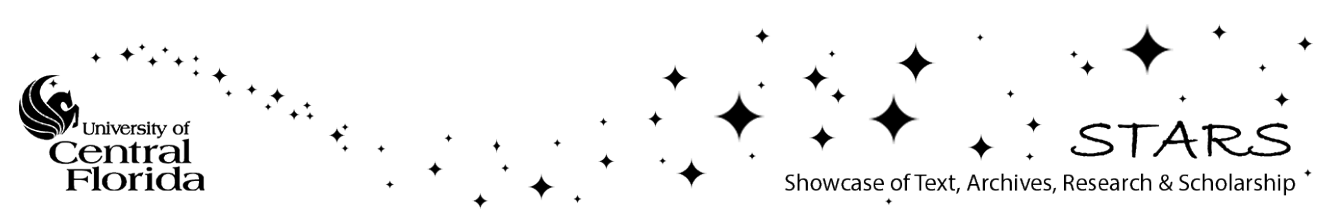




\title{
Imaging of Ankle Replacements
}

\author{
Gary M. LiMarzi, MD ${ }^{1} \quad$ Dzmitry M. Fursevich, MD ${ }^{1} \quad$ J. Dean Cole, MD ${ }^{2}$ Christopher W. Wasyliw, MD ${ }^{1}$ \\ Laura W. Bancroft, MD ${ }^{1,3}$
}

${ }^{1}$ Department of Radiology, Florida Hospital, Orlando, Florida

${ }^{2}$ Florida Hospital Orthopedic Institute, Orlando, Florida

3 University of Central Florida College of Medicine, Orlando, Florida

Address for correspondence Laura W. Bancroft, MD, Department of Radiology, Florida Hospital, 601 E. Rollins, Orlando, FL 32803 (e-mail: Laura.bancroft.md@flhosp.org).

Semin Musculoskelet Radiol 2015;19:67-76.
Abstract
Keywords
- ankle arthroplasty
- ankle replacement
- INBONE
- loosening
- osteolysis
- osteotomy

Significant innovations in the field of total ankle replacement surgery have occurred since the early disappointing experiences with first-generation devices. In recent years, total ankle arthroplasties are commonly placed in patients with end-stage ankle osteoarthrosis, supplanting ankle arthrodesis as the procedure of choice at some institutions. Careful clinical and preoperative radiographic evaluation is helpful to plan concomitant ligament release, ligament reconstruction, or osteotomy. Careful attention to radiographic signs of early hardware loosening or subsidence can prompt intervention while bone loss is still minimal, and computed tomography has an adjunct role in the evaluation of periprosthetic lucency and osteolysis.
Primary osteoarthrosis of the ankle is a rare condition, with an incidence $\sim 9$ times less than osteoarthrosis of the hip or knee. In contrast to other commonly replaced joints, total ankle arthroplasties (TAAs) are most commonly placed because of arthrosis related to prior trauma (-Fig. 1). ${ }^{1-6}$ The lower incidence of primary ankle osteoarthrosis may be related to a greater intrinsic resistance by the ankle articular cartilage to tensile and fracture stresses compared with the hip, implying greater resistance to routine wear and tear. For decades, the treatment of choice for end-stage ankle arthrosis was arthrodesis. ${ }^{5-9}$ Although joint fusion is commonly effective at alleviating ankle pain in the immediate term and can assist in the return to a more normal level of function and quality of life, there is a high incidence of longterm complications including ipsilateral hindfoot arthrosis, most commonly involving the subtalar joint. ${ }^{3,5,6,10}$ This association is likely due in large part to the altered gait biomechanics of a fused ankle with abnormal distribution of the lower extremity weightbearing axis. In the short term, complications of arthrodesis include nonunion, malunion, and infection. ${ }^{3,10}$

The ankle is a three-bone biomechanically complex joint that poses many unique challenges to effective joint salvage therapy. The ankle is unique in respect to other replaced joints because it must support up to five times body weight during normal ambulation but has decreased surface area compared with the knee or hip joint. ${ }^{2,4,9}$ Early ankle joint prostheses were pursued with great enthusiasm, energized by the successes of hip and knee replacement surgery. First-generation ankle arthroplasty hardware required the use of cement fixation as well as excessive tibial bone resection, which, combined with a poor reproduction of normal ankle mechanics, resulted in high early failure rates. ${ }^{2-4,6,10,11}$ Early surgical technique also largely ignored the importance of soft tissue rebalancing, contributing to poor outcomes. The large amount of bone resection increased the difficulty of salvage fusion surgery. For the next several years, ankle arthroplasty was largely abandoned, and arthrodesis remained the preferred procedure. ${ }^{10}$

In recent years, a renewed interest in ankle joint replacement surgery has developed, fueled in part by the continued improvement in hip and knee arthroplasties. Dissatisfaction with the long-term outcomes of arthrodesis and high rates of secondary hindfoot arthrosis fostered a new generation of design. 3,10 The designs used in second-generation ankle prostheses attempt to better emulate natural articular motion and require a smaller total volume of bone resection during placement. ${ }^{4,10}$ Routine postoperative follow-up includes serial clinical and radiographic evaluation to assess for the development of complications such as infection,
Issue Theme Imaging of Joint Replacements; Guest Editor, Theodore T. Miller, MD, FACR
Copyright (c) 2015 by Thieme Medical Publishers, Inc., 333 Seventh Avenue, New York, NY 10001, USA. Tel: +1(212) 584-4662.
DOI http://dx.doi.org/ 10.1055/s-0034-1396769. ISSN 1089-7860. 


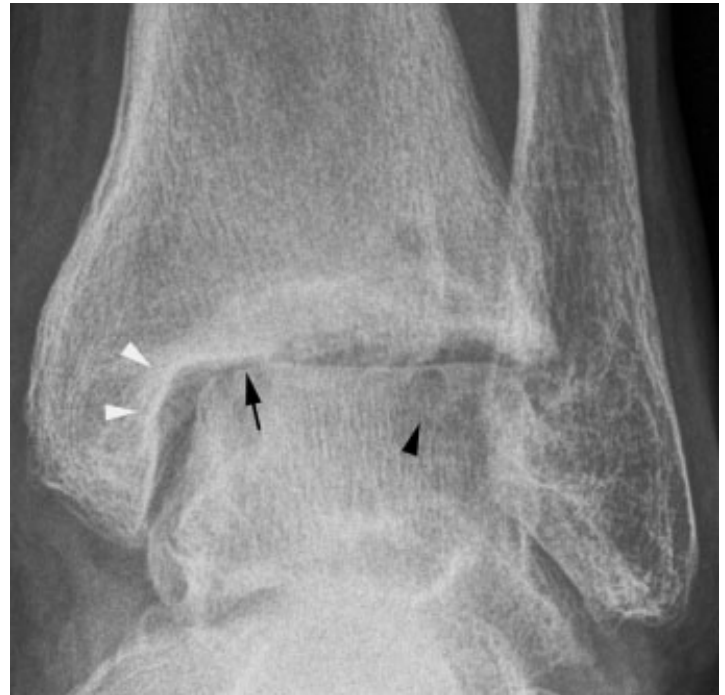

Fig. 1 Advanced ankle arthropathy in a 54-year-old man following a bimalleolar fracture. There is near-complete loss of the medial joint space (arrow) with subchondral sclerosis (white arrowheads) on anteroposterior radiograph. Also note subtle subarticular cystic change (black arrowhead) that may be more extensive than its radiographic appearance suggests.

hardware subsidence and malalignment, periprosthetic lucency, and component loosening. ${ }^{10}$

\section{Ankle Implant Design}

The ankle consists of superior, lateral, and medial articulations. The tibial plafond and talar dome articulate superiorly, and the lateral talus and lateral malleolus articulate laterally. The tibia, medial malleolus, and medial talar facet compose the medial articulation. ${ }^{7}$ Modern ankle arthroplasties are designed to better reproduce the natural motion of the joint during plantarflexion and dorsiflexion, and many implant designs also allow multiplanar motion including internal/ external rotation and inversion/eversion. Modern surgical technique emphasized the importance of ligament balancing to improve the loading pattern over the implant surface, increasing survivorship., 4

First-generation cemented implants had poor long-term outcomes. Patient satisfaction varied widely from 19 to 81\%, with lower satisfaction scores reported by patients with longer follow-up. ${ }^{6,7}$ Rates of loosening (as determined on postoperative radiographs) were high, ranging from 22 to 75\%. Factors implicated included the use of cement fixation, highly constrained designs, and the large amount of bone resection required for these devices.

Current generation implants are classified as either twoor three-component designs, referring to the respective absence or presence of a mobile polyethylene insert. In two-component designs, the tibial articular component and the polyethylene insert are a single fixed piece without an articulation at the component-polyethylene interface. These are commonly known as fixed-bearing implants. Three-component (mobile-bearing) designs have two articulations: one at the interface between the tibial component and polyethylene insert and the second at the insert-talar component interface. Three-component designs improve congruency of the implant, require less bone resection, and allow improved multiplanar motion. However, mobile-bearing components introduce the possibility of polyethylene dislocation and require that the implant fit tightly within the joint space. ${ }^{10}$ Implant designs differ in their relative degrees of conformity and constraint. Conformity describes the extent of contact between articular components, with high-conformity designs theoretically reducing component wear by reducing pointcontact stress along the joint. Mobile-bearing implants are fully conforming, whereas fixed-bearing devices are only partially conforming. Constraint refers to the extent to which a device resists motion in a particular direction. Highly constrained designs offer the benefit of improved joint stability, but they are associated with transfer of shear and rotatory forces at the component-bone interface, increasing the risk of loosening. ${ }^{8}$

A variety of ankle arthroplasty designs are currently in clinical use worldwide. Examples of commonly used devices in the United States include the Agility Total Ankle System (DePuy, Warsaw, IN), STAR (Scandinavian Total Ankle Replacement, Waldemar Link, Hamburg, Germany), Salto-Talaris Anatomic Ankle Prosthesis (Tornier, Stafford, TS), and INBONE Total Ankle (INBONE Orthopedics, Boulder, CO). The Agility Ankle (-Fig. 2) is the most common device used in the United States and was the first device to receive approval by the U.S. Food and Drug Administration (FDA) prior to 2006. ${ }^{5,11,12}$ Since that time, new devices have been approved by the FDA including the Salto-Talaris, INBONE, and STAR systems. ${ }^{12}$ Although these implants are approved for use with cement fixation, they are almost universally used off-label and feature porous components for fixation by bone ingrowth at the tibial and talar surfaces. ${ }^{6-8}$

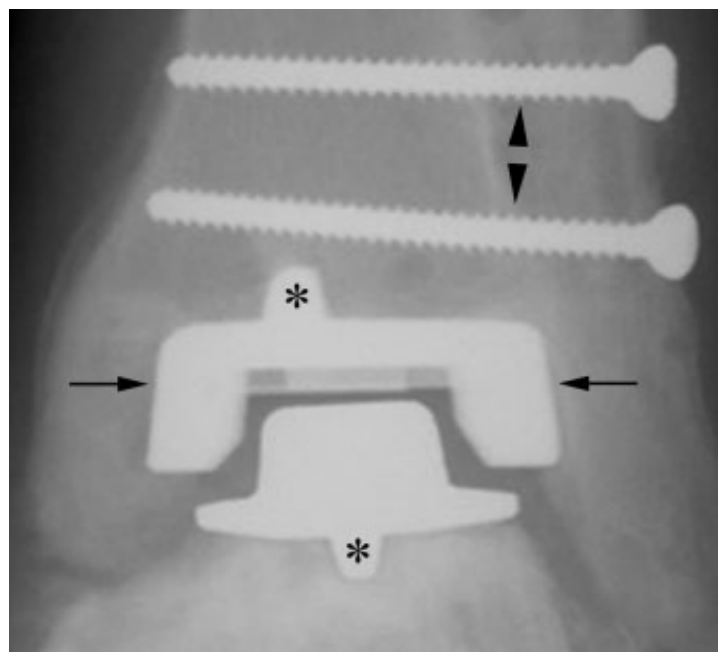

Fig. 2 Agility Total Ankle. This two-component fixed-bearing implant features a more constrained design with the large U-shaped tibial component (arrows) bracketing the talar component. Both components are anchored to the underlying bone with shallow vertical fins (asterisks). Syndesmotic fusion (arrowheads) accompanying the Agility implant enables improved distribution of weightbearing forces. 


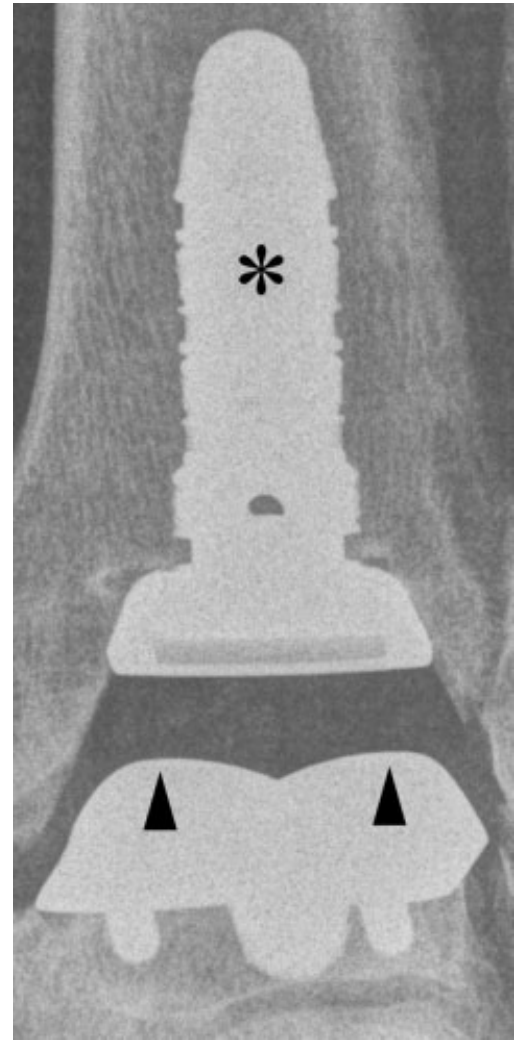

Fig. 3 INBONE ankle prosthesis. Anteroposterior radiograph delineates the two-component fixed-bearing device whose characteristic feature is its modular tibial stem (asterisk). There is a wide biconvex talar component (arrowheads) designed to improve tracking of the joint.

The most common two-component device is the Agility Ankle, introduced in 1984 and the longest used implant in the United States. As with all fixed-bearing implants, there is only partial conformity of the articulation. The tibial component is broad based and in contact with the tibial plafond and the bilateral malleoli. The talar component features a superior convexity that articulates with the tibial component in $\sim 22$ degrees of external rotation to better mimic normal ankle anatomy. ${ }^{8}$ Both the tibial and talar components are anchored by short fins. A unique feature of the Agility Ankle is syndesmotic fusion, performed to improve stability and reduce the risk of subsidence by increasing the surface area at the interface between the tibial component and bone. ${ }^{8}$

Another two-component design is the INBONE Total Ankle (-Fig. 3), with a unique modular anchoring system for fixation of the tibial component. ${ }^{8,12}$ This system can be adjusted to optimize bone contact in certain patients including those with fracture deformities. The INBONE system uses a proprietary guidance system to aid in proper intraoperative alignment of the components. ${ }^{8,13}$

The Salto Talaris Anatomic Ankle Prosthesis is a fixedbearing system characterized by a tapered fixation plug on the tibial component and requires a unique keyhole-shaped window in the anterior tibial cortex during installation. The talar component features a superior convexity with two lateral runners and a central depression to improve tracking of the polyethylene insert. ${ }^{8}$

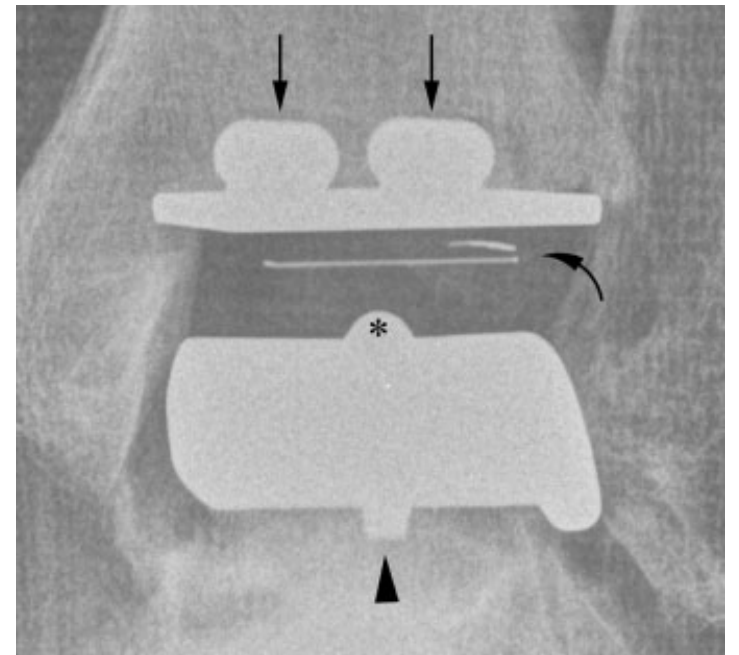

Fig. 4 Anatomy of the STAR ankle prosthesis. The STAR is a threecomponent mobile-bearing device with characteristic cylindrical bars (arrows) anchoring the flat tibial component. The convex talar component is anchored to the remaining talus with a vertical fin (arrowhead). A longitudinal ridge extending from the talar component assists with tracking during dorsiflexion and plantarflexion (asterisk). The mobile polyethylene insert contains radiopaque markers to aid evaluation (curved arrow).

The STAR prosthesis (-Fig. 4) is a mobile-bearing design that has been in use since the 1980s. The design consists of a flat tibial trapezoidal plate anchored by two cylindrical bars extending anteroposteriorly. This plate articulates with the polyethylene insert, which in turn articulates with the convex talar component featuring a longitudinal ridge that guides plantarflexion and dorsiflexion. The talar component is anchored to the talar body by a small finlike projection. ${ }^{8}$ All mobile-bearing devices feature a radiologic marker in the polyethylene insert to improve detection of dislocation or fracture postoperatively. 3

\section{Preoperative Evaluation}

Routine preoperative radiographs are essential in all patients undergoing TAA to evaluate the degree of ankle arthropathy as well as any signs of malalignment, particularly coronal plane deformities. Weightbearing anteroposterior (AP), later$\mathrm{al}$, and mortise projections are routinely obtained. ${ }^{1}$ Suboptimal alignment leads to abnormal weight distribution, increasing contact stresses and edge loading on the prosthesis, which accelerates polyethylene wear. ${ }^{14}$ Radiographic assessment is also necessary to identify surgical contraindications including avascular necrosis, advanced bone loss, and severe peripheral vascular disease. ${ }^{4,10}$ Careful radiographic assessment is critical because undiagnosed and uncorrected alignment issues increase the risk of postoperative failure. ${ }^{4,15}$

Coronal plane deformities are either valgus or varus, with varus deformities predominating. ${ }^{10,14}$ The distal tibial angle is formed by the intersection of two lines along the axes of the tibial diaphysis and tibial plafond on AP view. The proximal talar angle is formed by intersection of the axes along the superior talar surface and tibial diaphysis. The degree of 


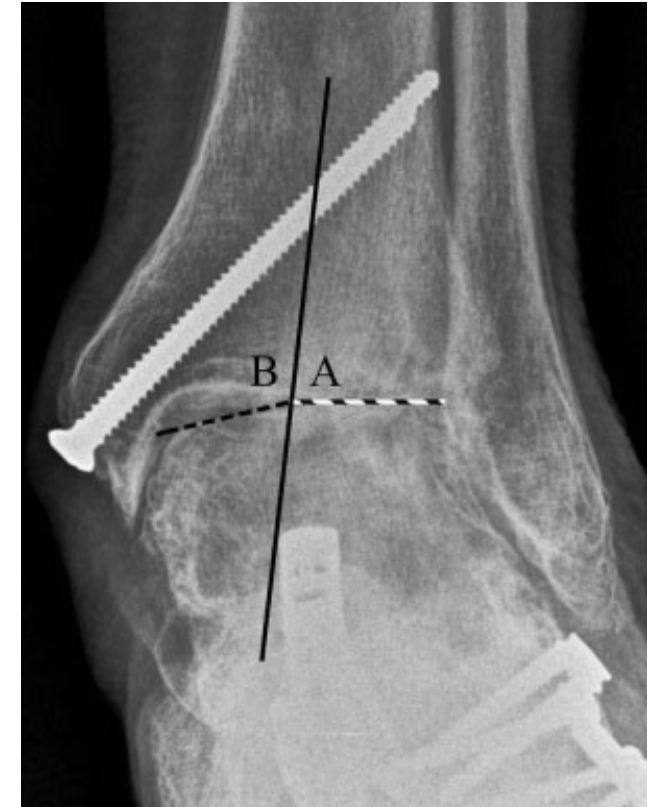

Fig. 5 Advanced ankle osteoarthrosis and valgus coronal plane deformity on preoperative radiograph. The degree of coronal-plane deformity is the difference between the distal tibial angle $(A)$ and the proximal talar angle (B).

coronal plane deformity is the difference between these two angles (-Fig. 5). ${ }^{1,10,14}$ In most cases, when the preoperative varus or valgus angulation $>30$ degrees, arthrodesis is recommended in place of arthroplasty. ${ }^{4}$ Furthermore, a coronal angulation deformity of $\geq 15$ degrees has been cited as a relative contraindication for TAA. ${ }^{4,8,10}$ In a prospective matched cohort study of 36 ankles with $\geq 10$ degrees of coronal varus deformity, Trajkovski and colleagues demonstrated no significant difference in patient-reported outcomes up to 35 months following TAA compared with patients with $<10$ degrees varus deformity. ${ }^{14}$

Ankle joint congruency is assessed on AP radiographs by extending lines along the tibial plafond and superior talar surface, and these axes should normally be parallel. ${ }^{10}$ The tibial slope is measured on lateral radiographs and is the intersection of lines drawn along the posterior tibial cortex and the anterior and posterior tibial articular surface. ${ }^{1}$ Lateral views of the tibia may be useful to look for recurvatum or procurvatum deformities because these alter the location of the ankle in relation to the weightbearing axis of the leg and should be addressed before placement of the prosthesis. ${ }^{10}$

When evaluating the extent of ankle arthropathy on preoperative radiographs, particular attention should be paid to the degree of subchondral cystic change in the talar dome and tibial plafond. On occasion, the size of these cysts may be underestimated on radiography, and cystic degeneration may be severe enough to compromise the bone's ability to support the implanted hardware. ${ }^{10,15}$ The presence of bulky osteophytes may obscure significant detail on radiography. In questionable cases, computed tomography (CT) can be helpful in more accurately assessing the degree of cystic change. $^{10,15}$

\section{Postoperative Evaluation}

Serial postoperative radiographic evaluation in conjunction with clinical assessment is routine in following patients with TAA. Because early postoperative complications can present with subtle changes, attention to proper imaging technique and positioning is critical. The age of the implant must be known because expected complications occur at different postoperative times. Weightbearing views (with a minimum of AP and lateral projections provided) should be compared with all prior studies available. Correlation with prior studies is very important to detect subtle changes in implant positioning that may suggest early hardware loosening or subsidence. ${ }^{8}$

Postoperative ankle alignment is determined by using several linear and angular measurements. Although these measurements vary by implant type, the general principles remain constant. The most commonly implanted TAAs at our institution are the Agility Total Ankle and the STAR ankle prosthesis, and our evaluation of linear and angular alignment is based on these models. For STAR implants, tibial component angle is assessed by drawing a line along the axis of the tibial diaphysis on the AP view that intersects a line extending along the horizontal axis of the tibial component. This is commonly referred to as the $\alpha$ angle and reflects the degree of coronal varus or valgus deformity; neutral alignment should measure 90 degrees (-Fig. 6a)..$^{8,10,16,17}$ Drawing the same lines on the lateral projection produces the $\beta$ angle, which represents the amount of dorsiflexion or plantarflexion of the tibial component ( - Fig. $\mathbf{6 b}$ ). As with the $\alpha$ angle, the $\beta$ angle should equal 90 degrees when alignment is neutral. The gamma angle is measured by the intersection of a line paralleling the long axis of the talar component with a line drawn from the posterior aspect of the talar component through the middle of the talar neck (-Fig. 6b). ${ }^{8,10,16} \mathrm{~A}$ change in angulation $>5$ degrees in any of these measurements is suspicious for component migration. ${ }^{8,17,18}$

Multiple linear measurements can be used to assess for STAR implants in cases of suspected hardware subsidence or migration. ${ }^{8,16}$ Subsidence of the tibial component is detected on AP images by measuring the distance between the base of the tibial prosthesis and the transmalleolar line (connecting the tips of the medial and lateral malleoli). ${ }^{8,16}$ Talar subsidence is evaluated on lateral radiographs using a reference line drawn through the long axis of the talar component and a line intersecting the calcaneal tubercle and dorsal aspect of the talonavicular joint. ${ }^{8,16}$ Vertical lines are then drawn extending from the anterior and posterior edges of the talar component, and the distance between these points and the reference line are measured (-Fig. 7). ${ }^{8,16}$ In this way, the degree of talar subsidence can be monitored over serial imaging examinations. Loosening of the talar component is suggested when there is $>5 \mathrm{~mm}$ of subsidence on the lateral view. ${ }^{1,8,16,18}$ In addition, because the STAR arthroplasty is a mobile-bearing device, it is susceptible to displacement and/or fracture of the polyethylene insert.

Agility TAA radiographic alignment and positioning measurements are similar to other devices. ${ }^{17}$ Angular measurements used to determine the relative positioning of the tibial component are identical to those applied to patients with the 
a
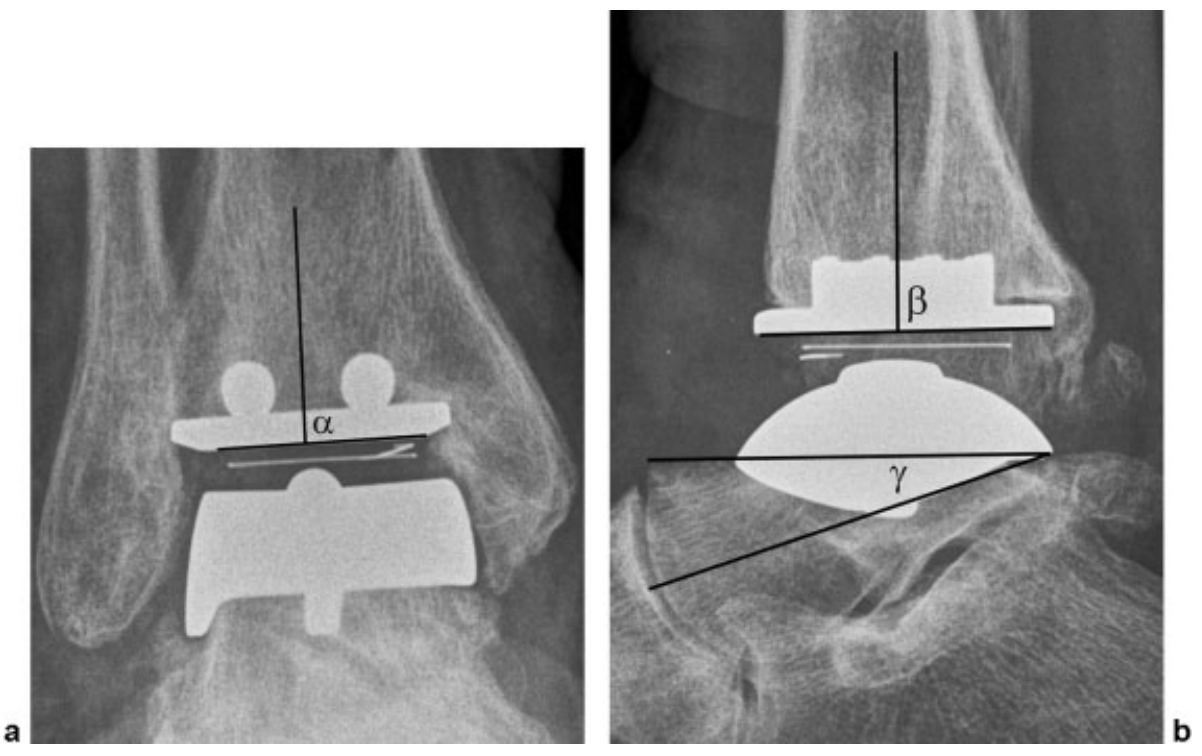

Fig. 6 Alignment of the STAR ankle prosthesis. Alignment of the tibial component is determined by the $\alpha$ and $\beta$ angles on the (a) anteroposterior and (b) lateral views, respectively. Talar component alignment is evaluated with the gamma ( $\mathrm{Y}$ ) angle. A change of $\geq 5$ degrees on serial examinations is suspicious for hardware migration.

STAR or INBONE prostheses. One difference is measurement of the talar component angle, derived from the intersection of a line drawn along the inferior aspect of the talar implant with a line paralleling the floor: Ellington and colleagues reported an average of 3.9 degrees of extension in their series, with a wide range of 20 degrees of extension to 10 degrees of flexion (-Fig. 8). ${ }^{17}$ Subsidence of the talar component is suggested by altered positioning of the component. Ellington et al reported their novel classification for subsidence relative to the subtalar joint, although the subtalar joint is often hard to delineate on radiographs depending on patient positioning: (1) Grade 1 is no subsidence; (2) grade 2 is subsidence that is not to the level of the subtalar joint; and (3) grade 3 is subsidence to the level of or inferior to the subtalar joint. ${ }^{17}$

Postoperative evaluation of patients with the INBONE ankle prosthesis shares many of the general principles used for
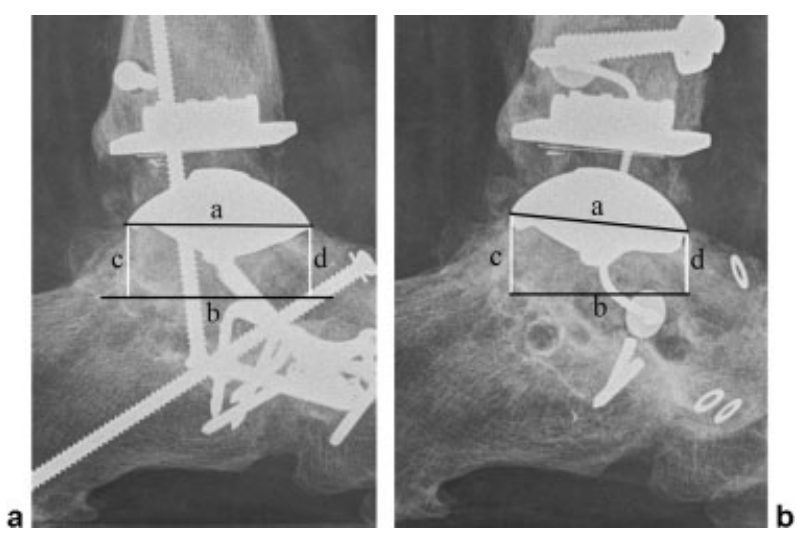

Fig. 7 Evaluating talar component subsidence. A line drawn through the long axis of the talar component (a) parallels the reference line drawn along the floor (b). Posterior (c) and anterior (d) vertical lines should be approximately equal length. Subsidence occurs when there is a $>5-\mathrm{mm}$ change in hardware positioning on serial examinations. assessing the STAR implant, with slight differences in determining hardware positioning. The talocalcaneal angle is formed by the intersection of a line drawn along the inferior edge of the talar dome component with a line extending through the superior aspect of the talonavicular joint, and it should be $\sim$ 12.5 degrees ( - Fig. 9a). ${ }^{1}$ Measurement of the tibial component angle on the AP ( $\alpha$ angle) and lateral ( $\beta$ angle) projections is identical to the method used for STAR or Agility ankles and should be $\sim 90$ degrees. ${ }^{1,8}$ The talar component angle is formed by a line paralleling the flat surface of the talar component intersecting a line connecting the anterior edge of the talar component with the inferior aspect of the posterior talus; the normal angle is $\sim 10$ degrees ( $\mathbf{F i g . ~ 9 b}$ ). ${ }^{1}$ Linear measurements

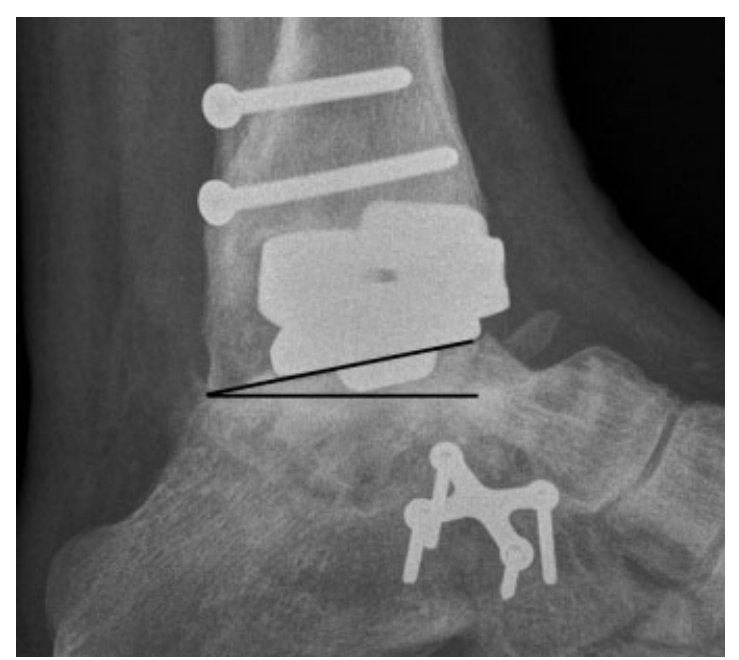

Fig. 8 Talar component angle measurement for the Agility Total Ankle. The angle is formed by the intersection of a line along the inferior edge of the talar component with a line paralleling the floor on standing lateral radiograph. Changes in this angle over serial evaluations suggest talar subsidence. 

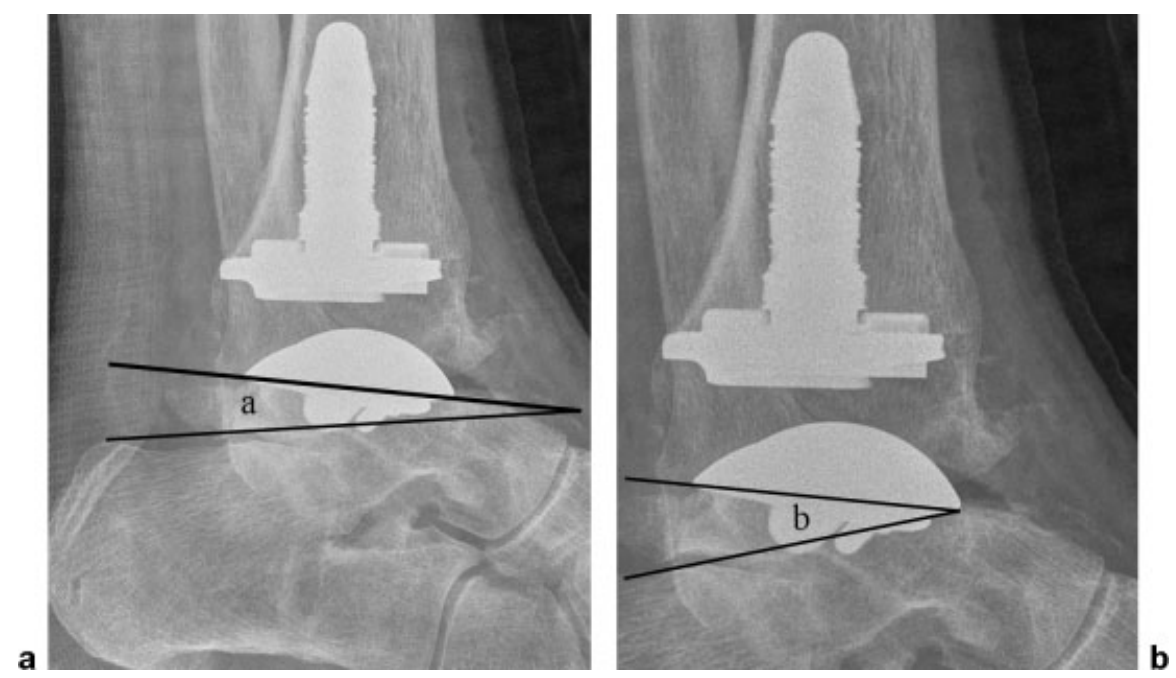

Fig. 9 Measuring the (a) talocalcaneal and (b) lateral talar component angles in the INBONE ankle prosthesis. Changes in these angles over serial examinations are concerning for hardware subsidence.

in patients with the INBONE ankle prosthesis are virtually identical to those used in the STAR or Agility ankles with regard to evaluating for hardware subsidence or loosening. ${ }^{1}$

In addition to changes in hardware position and alignment, prosthetic loosening can be suggested by the development of periprosthetic lucency at the component-bone interface. ${ }^{8,19}$ In one series, the incidence of any periprosthetic lucency, regardless of clinical significance, was reported at 98\%. ${ }^{17}$ Careful attention is required to differentiate early osteolysis from benign periprosthetic lucencies measuring $<2 \mathrm{~mm}$ in width. In normal patients with the STAR implant, thin linear lucencies may form at the tibial component-bone interface within 6 months of surgery. This is thought to be the result of stress shielding due to the more proximal anchoring of the tibial barrels. ${ }^{16}$ Motion occurring at the tibiofibular syndesmosis can lead to friction between the component and medial aspect of the distal fibula, evident as a focal linear lucency between the tibial component and medial surface of the lateral malleolus. In a series of 84 patients treated with the STAR prosthesis, Mann and colleagues identified this radiographic finding in $11 \%$ of their patients, and this lucency measured $2 \mathrm{~mm}$ on average (range: $1-5 \mathrm{~mm}$ ). ${ }^{16}$ These authors also reported that neither stress shielding nor osteolysis along the medial aspect of the lateral malleolus is associated with an increased risk of hardware loosening. ${ }^{16}$ It is also important not to confuse iatrogenic lucency, such as those related to predrilling for the placement of tibial barrels with those caused by loosening (-Fig. 10). Drilling defects are evident on the immediate postoperative radiographs and will resolve over time as they fill with bone. ${ }^{8}$ Developing lucencies $>2 \mathrm{~mm}$, however, are highly concerning for aseptic hardware loosening. Radiography may be limited in its ability to fully assess the extent of osteolysis, and CT can more accurately visualize the degree of bone loss ( $\mathbf{- F i g . ~ 1 1 )})^{5,8,20}$

The use of CT in the postoperative evaluation of ankle arthroplasties has received renewed interest in recent years due to improvements in the ability to minimize metal-related artifacts. $^{5}$ In the past, streak and beam-attenuation artifacts from the implanted hardware greatly impeded evaluation of surrounding structures, making the detection of subtle lucencies impossible. ${ }^{5,8}$ To reduce beam attenuation and streak artifact from the implanted metal, the tube voltage should be increased to $140 \mathrm{kVp}$ and the tube current should be raised to 200 mAs. ${ }^{5,8,19}$ Increasing the energy of the photon beam reduces scatter and aids penetration and visualization of the anatomy adjacent to the metal hardware. Iterative reconstruction algorithms are also utilized with metallic implants to increase insensitivity to noise and provide an optimal image in the case of incomplete data. ${ }^{19}$ Image thickness $<2 \mathrm{~mm}$ is also helpful due to improved spatial resolution, although this comes at the cost of increased image noise. ${ }^{5}$ Careful alignment of the implant relative to the photon beam can also help reduce metal artifact by ensuring that the smallest possible

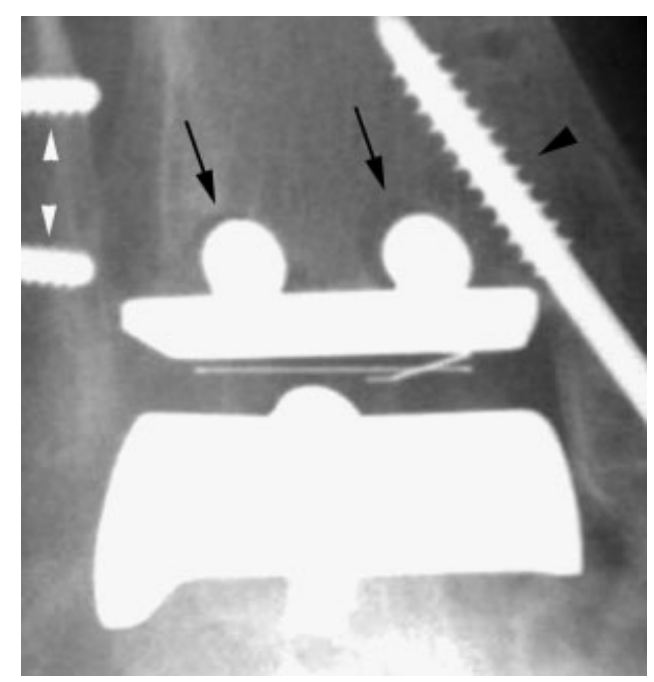

Fig. 10 Predrilled holes for cylindrical tibial anchors of the STAR ankle prosthesis. Anteroposterior radiograph shows the normal appearance of predrilled holes (arrows) that should not be confused for osteolysis from particle disease or hardware loosening. Note fixation hardware for intraoperative fractures of the medial malleolus (black arrowhead) and distal fibula (white arrowheads). 

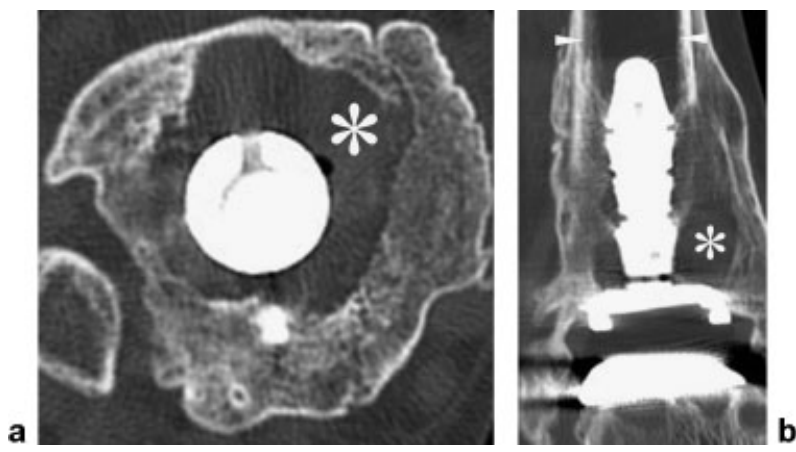

Fig. 11 Particle disease related to INBONE ankle prosthesis. (a) Axial and (b) coronal computed tomography images in a patient with the INBONE ankle prosthesis show extensive periprosthetic lucency surrounding the tibial component stem with some bone integration along the proximal aspect of the stem as well as tilt of the tibial articular surface, consistent with particle disease. Tibial reaming (arrowheads) prior to tibial stem placement should not be confused with osteolysis.

cross section of the hardware is traversed by the beam. ${ }^{8} \mathrm{CT}$ can also allow better visualization of the polyethylene component in mobile-bearing devices in cases of suspected dislocation or fracture ( $\mathbf{- F i g . ~ 1 2 ) . ~}{ }^{8}$

\section{Outcomes}

When compared with the poor outcomes seen in patients with first-generation ankle arthroplasties, the intermediate- and long-term outcomes seen with modern prostheses have been promising. Long-term results of the STAR ankle have been reported with a 5-year implant survival of $96 \%$, decreasing to $90 \%$ survival at 10 years. ${ }^{16}$ These results are concordant with intermediate-term results published by Wood and colleagues, reporting a 5-year survival of 93\%.,16,21 However, the rate of radiographic complications (periprosthetic lucency $>2 \mathrm{~mm}$, subsidence, periprosthetic fracture, syndesmotic screw loosening, hardware fracture, heterotopic ossification, increased

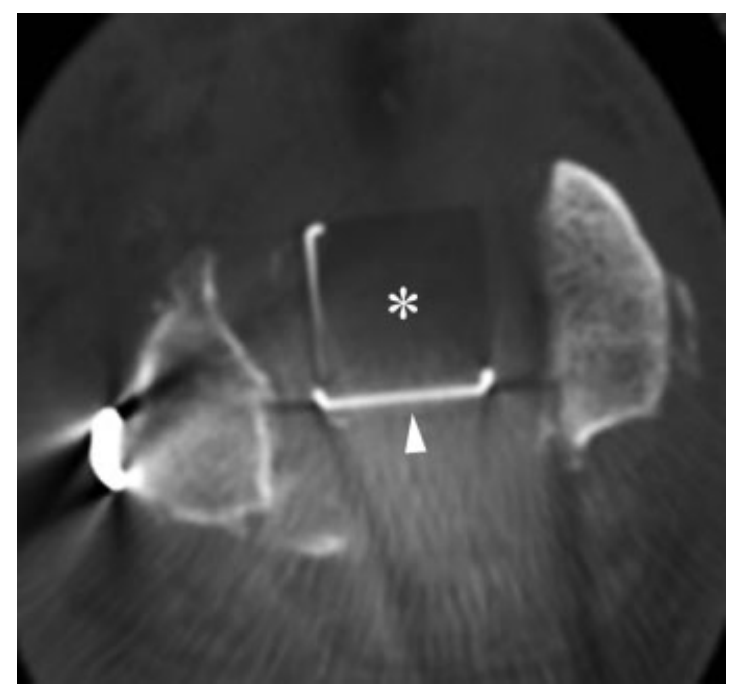

Fig. 12 Proper positioning of the polyethylene insert in a patient with a mobile-bearing total ankle arthroplasty. The polyethylene tibial insert (asterisk) is centered in the ankle joint without evidence of fracture or migration. The metallic marker within the periphery of the insert (arrowhead) is unique to mobile-bearing prostheses.

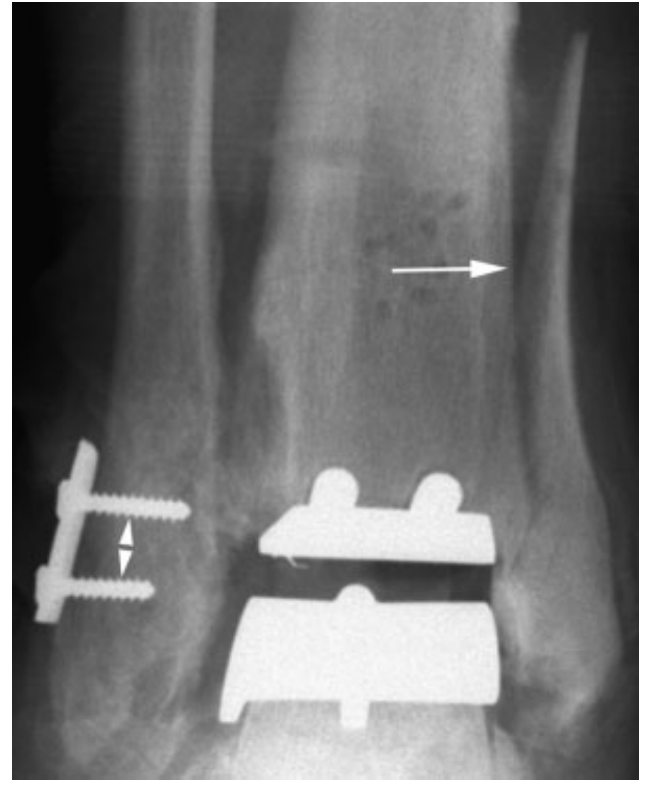

Fig. 13 Total ankle arthroplasty and medial malleolar osteotomy. Intraoperative fluoroscopic spot image of the right ankle during placement of a STAR ankle prosthesis demonstrates a rotated long oblique osteotomy through the medial malleolus and distal tibial shaft (arrow), with the purpose of preserving the medial shelf. Old transfixed distal fibular fracture (arrowheads) is noted.

varus or valgus angulation, ankle gutter narrowing, and syndesmotic nonunion or fracture) has been described to be as high as $62 \%$, although these findings do not necessarily correspond with hardware failure. ${ }^{2}$ Surgical revision rates between $14 \%$ and $32 \%$ have been reported across various implant types after 5 years, higher than reported for hip and knee revision arthroplasty rates. ${ }^{3,17}$ Criswell et al reported a revision rate of $39 \%$ after 8 years in a series of 42 ankles implanted with the Agility Total Ankle. The survival rate at 9 years was estimated between $47 \%$ and $77 \% .{ }^{11}$ Revision surgery may include placement of a new prosthesis, conversion to arthrodesis, osteotomy (-Fig. 13), ligament release/reconstruction, gutter debridement, or rarely below-the-knee amputation. ${ }^{11,17}$ Interestingly, the intermediate-term revision rates in patients with TAAs are twice as high as for patients treated with ankle fusion. This is attributed to the high complexity of total ankle revision arthroplasty compared with fusion as well as the relative inexperience of some surgeons in performing arthroplasties. ${ }^{22}$

\section{Complications}

Based on a systematic review, Glazebrook and colleagues proposed that complications be classified as high, intermediate, or low severity in respect to the likelihood of hardware failure. ${ }^{4,16,23,24} \mathrm{High}$-grade complications include deep infection and aseptic loosening. Medium-grade complications include technical errors during implantation and hardware subsidence. Low-grade complications consist mostly of intraoperative and postoperative fractures, as well as issues related to wound healing. 24

Initially, high-grade complications are associated with a $>50 \%$ chance of failure. Medium-grade complications carry 
a $<50 \%$ risk of failure, and low-grade issues are not associated with failure. ${ }^{23}$ However, a retrospective study by Gadd et al suggests that every complication (with the exceptions of intraoperative fracture and delayed wound healing) conferred a failure rate of at least $50 \%{ }^{24}$ Based on their results, Gadd proposed a revision of the three-grade model to only two grades: high and low. ${ }^{24}$ All high-grade complications carry a failure risk of $\geq 50 \%$, whereas low-grade complications do not confer a risk of failure and mostly include intraoperative fracture and impaired wound healing. ${ }^{24}$

When based on chronology, complications are defined as intraoperative, early postoperative, or delayed. Intraoperative complications include neurovascular and musculotendinous injury, improper hardware positioning, excess bone resection, and periprosthetic fractures. Fractures of the medial malleolus are the most commonly seen, with an estimated incidence of $20 \%{ }^{4,8,10,13}$ Early postoperative complications include wound infection, delayed surgical wound healing, and stress fractures of the medial malleolus. A complication unique to the Agility TAR is nonunion of the syndesmotic arthrodesis. ${ }^{4,8}$ Successful fusion of the syndesmosis is critical for stability of the tibial component because this allows improved weight distribution. ${ }^{4,7}$ Syndesmotic nonunion increases the risk of tibial component subsidence and osteolysis. ${ }^{4}$ When evaluating outcomes in the first 100 patients receiving the Agility prosthesis, Pyevich et al showed early

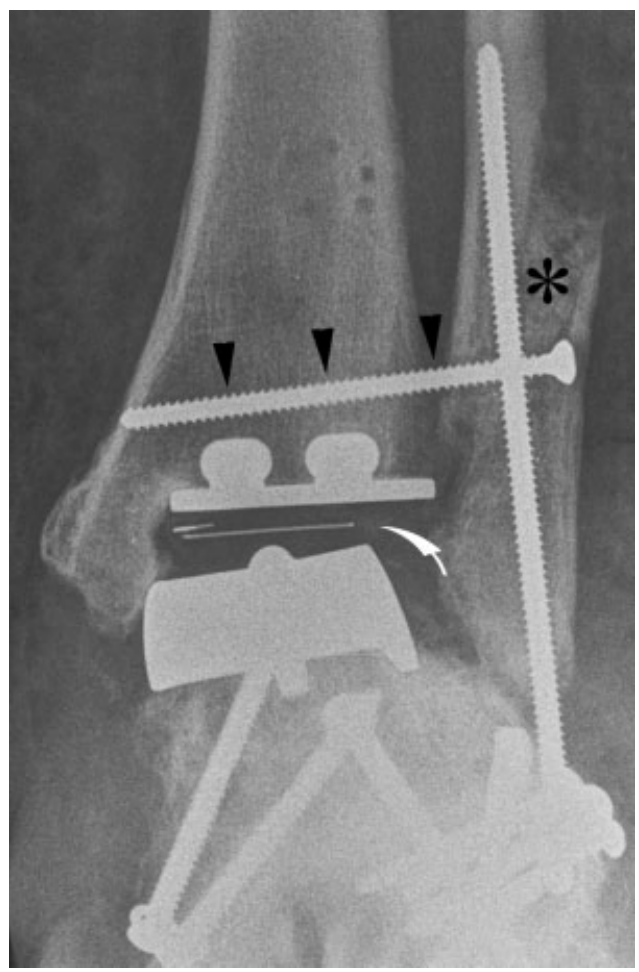

Fig. 14 Persistent coronal valgus deformity after varus-inducing fibular osteotomy. Postoperative anteroposterior radiograph in a 70year-old woman shows a persistent coronal valgus deformity despite a varus-inducing fibular osteotomy (asterisk). There is also syndesmotic cross-fixation for additional stability (arrowheads). Hardware fusion of the syndesmosis in $62 \%$ of patients by 6 months postoperatively. ${ }^{7,25}$ Although only $9 \%$ of patients experienced nonunion, most of the cases of tibial component migration occurred in this patient subset. ${ }^{7}$ More recent studies have described syndesmotic nonunion in only 0 to $2.7 \%$ of cases. ${ }^{2,17}$

Wound healing problems are reported in up to $10 \%$ of cases, often as a result of poor peripheral vascular supply. $3,8,10$ A $>12$ pack-year smoking history is considered a risk factor for nonhealing wounds. ${ }^{3}$ Surgical wound infection, fortunately, is relatively uncommon and reported in 4 to $6 \%$ of patients. $^{3,8,10}$ This incidence, however, is higher than what is reported for hip or knee replacements. ${ }^{4}$ When they do occur, however, infection of the hardware and deep soft tissues requires removal of the prosthesis, aggressive debridement, and placement of an antibiotic-impregnated spacer. ${ }^{4,8}$

Delayed postoperative complications are primarily related to problems with hardware alignment and support. Osteolysis with aseptic hardware loosening, hardware subsidence and migration, displacement or fracture of the polyethylene spacer in mobile-bearing devices, gutter impingement from bone overgrowth, and periprosthetic fractures are among the most commonly encountered delayed complications. ${ }^{8,10,19}$ Poor congruity of the tibial and talar components has been associated with a 10 times increased risk of developing edgeloading (-Figs. 14 and 15). ${ }^{4}$ Edge-loading of the hardware components occurs as a result of altered weightbearing in the

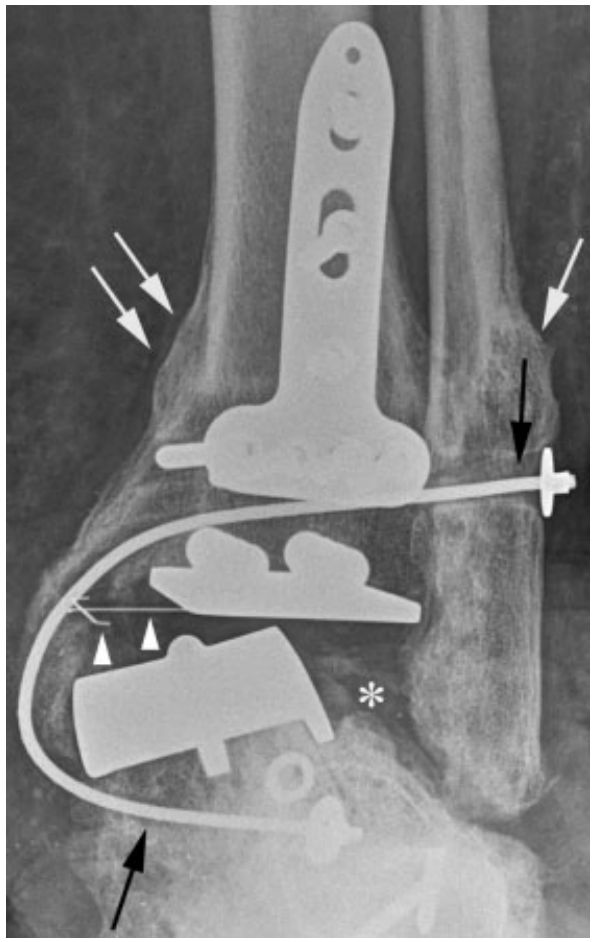

Fig. 15 Persistent coronal plane deformity after fibular and tibial osteotomies and deltoid ligament reconstruction. Anteroposterior radiograph shows coronal plane deformity despite both fibular and tibial osteotomies (white arrows) and reconstruction of the deltoid ligament with cable fixation (black arrows). There is subluxation of the ankle joint with medial displacement of the polyethylene insert (arrowheads) and incongruity of the tibial and talar components. Subtalar impingement from valgus deformity and lateral gutter osteophytes (asterisk) are additional complications. 
joint and is associated with altered component alignment over time. ${ }^{4,8,14}$ If ligamentous stability is not ensured at the time of implantation, progressive malalignment will increase the likelihood of edge-loading. ${ }^{8,10}$ The abnormal contact stresses that result will eventually cause polyethylene wear, leading to particle wear and osteolysis. ${ }^{4,8,14}$ Mobile-bearing designs are more apt to edge-loading because their motion is less constrained than fixed-bearing implants. ${ }^{8}$ Subtle, early changes can be detected by nonparallel axes along the articular surfaces of the tibial and talar components. ${ }^{10}$ Advanced edge-loading may be seen as widening of the medial or lateral gutters (reflecting eccentric wear on the polyethylene insert), which increases the risk for insert fracture or subluxation in the case of mobile-bearing devices. ${ }^{8,10} \mathrm{CT}$ can improve detection of subtle polyethylene fractures not appreciated on radiography.

Laxity of the medial or lateral collateral ankle ligaments can result in dislocation toward the weaker side. Varus deformities are more common, usually related to deltoid ligament insufficiency, and may require surgical tensioning or reconstruction. ${ }^{1}$ Postoperative measurements will reveal abnormal coronal varus or valgus alignment of the tibial and talar components, and there will be displacement of the radiographic marker within the insert. ${ }^{10}$ Coronal plane deformities within 5 degrees of neutral do not increase the risk of functional impairment or hardware failure. ${ }^{16}$ Surgical correction of coronal plane angulation deformities includes ligament release, reconstruction, or osteotomy.

Periprosthetic lucencies that are wider than $2 \mathrm{~mm}$ or progressive on serial imaging are worrisome for osteolysis from either particle disease or hardware loosening related to edge-loading of the polyethylene spacer. ${ }^{4,8,16}$ Ballooning osteolysis refers to a specific pattern of periprosthetic lucency along the lateral tibial component-bone interface. ${ }^{4,8}$ This is attributed to persistent micromotion and shear stress at this site; most cases stabilize within 1 year and are not associated with hardware failure (-Fig. 16). ${ }^{8}$ In the series by Lee and colleagues, periprosthetic lucencies of at least $2 \mathrm{~mm}$ were detected in $34 \%$ of cases. Of these, $89 \%$ were detected around the tibial component; only $11 \%$ involved the talus. ${ }^{2}$ In a series of 114 STAR implants, only 6\% required surgical revision because of aseptic loosening. ${ }^{3}$ This rate is much lower than had been reported in earlier studies, in which revision rates for aseptic loosening were between $24 \%$ and $34 \%{ }^{8}$ The combination of periprosthetic osteolysis and hardware subsidence is associated with higher rates of symptomatic ankle instability when compared with either entity alone, and this subset of patients has a significantly higher rate of revision surgery. 2,19

Because most TAA designs retain some amount of the medial and lateral articular surfaces of the ankle, there is a risk of developing marginal osteophytes., ${ }^{3,10,26}$ Over time, these osteophytes may become large enough to cause functional impairment and pain as a result of medial or lateral gutter impingement. ${ }^{3,10}$ Contributing factors are thought to include poorly sized hardware components, uncorrected coronal plane deformity, aseptic loosening, talar subsidence, and residual intraarticular debris. ${ }^{26}$ Symptomatic gutter im-

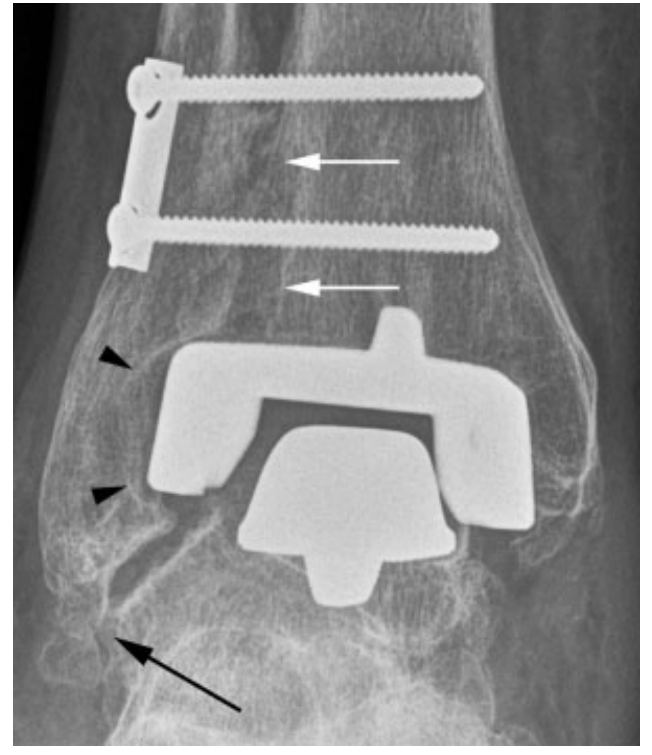

Fig. 16 Lateral gutter impingement by bulky osteophytes (black arrow) in a 79-year-old woman with Agility ankle prosthesis. Fibular osteolysis adjacent to the lateral edge of the tibial component (arrowheads) is due to mechanical forces at the component-bone interface in the initial postoperative period. Distal syndesmotic arthrodesis was also performed (white arrows).

pingement has been referenced as one of the most common delayed postoperative complications requiring surgical revision. ${ }^{3,8}$ Serial radiographs show progressive obliteration of the gutters by bulky osteophytes, and fractures may result when there is osseous bridging across joint spaces. ${ }^{26}$

In a series of patients with the Agility arthroplasty, symptomatic gutter impingement requiring debridement was the most common complication, occurring in $18.9 \%$ of cases by 31 months postoperatively. ${ }^{26}$ In contrast, gutter debridement was required in $16 \%$ of patients receiving the INBONE prosthesis and only $9 \%$ of patients with the STAR ankle. ${ }^{26}$ Lee et al reported an even lower incidence, in which only $5.4 \%$ of patients developed symptomatic gutter narrowing during a similar follow-up period. ${ }^{2}$ In the series by Schuberth, the INBONE and STAR systems were more likely to develop symptomatic gutter impingement within 19 months and 10 months, respectively, significantly earlier than the Agility replacement system. The authors attributed this phenomenon to the greater risk of talar component subsidence in the Agility ankle, with subsequent development of a more indolent pattern of osteophyte formation. ${ }^{26}$

Subsidence of the tibial and talar components is defined as a positional change $>5 \mathrm{~mm}$ on serial postoperative radiographs. The risk of subsidence is increased in cases of severe subchondral cystic change that can lead to weakened component support and poor bony ingrowth. ${ }^{1,8,10}$ In a series by Lee et al, subsidence was identified in $24.4 \%$ of the 197 implants evaluated that included both Agility and STAR devices ${ }^{2}$ Ellington and colleagues studied postoperative complications in the Agility Total Ankle and reported an increased rate of surgical revision in patients with worsening talar subsidence. ${ }^{17}$ In another study of INBONE ankle replacements, 
talar subsidence was noted in all cases of hardware failure. ${ }^{1}$ This highlights the importance of the lateral talar angle on postoperative imaging of the INBONE prosthesis because this measurement depends on talar positioning and height. ${ }^{1}$

In conclusion, there have been significant innovations in the field of total ankle replacement surgery since the early disappointing experiences with first-generation devices. In recent years, TAAs are commonly placed in patients with endstage ankle osteoarthrosis, supplanting ankle arthrodesis as the procedure of choice at some institutions. Pre- and postoperative radiographs are helpful in evaluating patients with ankle prostheses, and the radiologist must have sufficient knowledge of the normal imaging appearance and the myriad of potential complications to provide a quality, meaningful interpretation. Accurate description of coronal plane deformities on preoperative imaging allows surgical correction at the time of implantation with ligament release, reconstruction, or osteotomy. Careful attention to radiographic signs of early hardware loosening or subsidence can prompt intervention while bone loss is still minimal, and CT has a proven adjunct role in the evaluation of periprosthetic lucency and polyethylene migration. Finally, long-term survival rates with modern devices have been encouraging, and radiologists should be prepared to encounter more of these patients in the near future.

\section{References}

1 Datir A, Xing M, Kakarala A, Terk MR, Labib SA. Radiographic evaluation of INBONE total ankle arthroplasty: a retrospective analysis of 30 cases. Skeletal Radiol 2013;42(12):1693-1701

2 Lee AY, Ha AS, Petscavage JM, Chew FS. Total ankle arthroplasty: a radiographic outcome study. AJR Am J Roentgenol 2013;200(6): 1310-1316

3 Noelle S, Egidy CC, Cross MB, Gebauer M, Klauser W. Complication rates after total ankle arthroplasty in one hundred consecutive prostheses. Int Orthop 2013;37(9):1789-1794

4 DiDomenico LA, Anania MC. Total ankle replacements: an overview. Clin Podiatr Med Surg 2011;28(4):727-744

5 Hanna RS, Haddad SL, Lazarus ML. Evaluation of periprosthetic lucency after total ankle arthroplasty: helical CT versus conventional radiography. Foot Ankle Int 2007;28(8):921-926

6 Raikin SM, Rasouli MR, Espandar R, Maltenfort MG. Trends in treatment of advanced ankle arthropathy by total ankle replacement or ankle fusion. Foot Ankle Int 2014;35(3):216-224

7 Saltzman CL, McIff TE, Buckwalter JA, Brown TD. Total ankle replacement revisited. J Orthop Sports Phys Ther 2000;30(2): 56-67
8 Bestic JM, Bancroft LW, Peterson JJ, Kransdorf MJ. Postoperative imaging of the total ankle arthroplasty. Radiol Clin North Am 2008;46(6):1003-1015, v-vi

9 Labib SA, Raikin SM, Lau JT, et al. Joint preservation procedures for ankle arthritis. Foot Ankle Int 2013;34(7):1040-1047

10 Cadden AR. Imaging in total ankle replacement. Semin Musculoskelet Radiol 2012;16(3):205-216

11 Criswell BJ, Douglas K, Naik R, Thomson AB. High revision and reoperation rates using the Agility ${ }^{\mathrm{TM}}$ Total Ankle System. Clin Orthop Relat Res 2012;470(7):1980-1986

12 Greenfield S, Ellis S. The bright future for total ankle replacements. Curr Rev Musculoskelet Med 2013;6(4):273-275

13 Abicht BP, Roukis TS. The INBONE II Total Ankle System. Clin Podiatr Med Surg 2013;30(1):47-68

14 Trajkovski T, Pinsker E, Cadden A, Daniels T. Outcomes of ankle arthroplasty with preoperative coronal-plane varus deformity of $10^{\circ}$ or greater. J Bone Joint Surg Am 2013;95(15):1382-1388

15 Steck JK, Anderson JB. Total ankle arthroplasty: indications and avoiding complications. Clin Podiatr Med Surg 2009;26(2): 303-324

16 Mann JA, Mann RA, Horton E. STAR ${ }^{\mathrm{TM}}$ ankle: long-term results. Foot Ankle Int 2011;32(5):S473-S484

17 Ellington JK, Gupta S, Myerson MS. Management of failures of total ankle replacement with the agility total ankle arthroplasty. J Bone Joint Surg Am 2013;95(23):2112-2118

18 Preyssas P, Toullec É, Henry M, Neron JB, Mabit C, Brilhault J. Total ankle arthroplasty-three-component total ankle arthroplasty in western France: a radiographic study. Orthop Traumatol Surg Res 2012;98(4, Suppl): S31-S40

19 Kohonen Ia, Koivu H, Vahlberg T, Larjava H, Mattila K. Total ankle arthroplasty: optimizing computed tomography imaging protocol. Skeletal Radiol 2013;42(11):1507-1513

20 Kohonen Ia, Koivu H, Pudas T, Tiusanen H, Vahlberg T, Mattila K Does computed tomography add information on radiographic analysis in detecting periprosthetic osteolysis after total ankle arthroplasty? Foot Ankle Int 2013;34(2):180-188

21 Wood PL, Deakin S. Total ankle replacement. The results in 200 ankles. J Bone Joint Surg Br 2003;85:334-341

22 Daniels TR, Younger ASE, Penner $\mathrm{M}$, et al. Intermediate-term results of total ankle replacement and ankle arthrodesis: a COFAS multicenter study. J Bone Joint Surg Am 2014;96(2):135-142

23 Glazebrook MA, Arsenault K, Dunbar M. Evidence-based classification of complications in total ankle arthroplasty. Foot Ankle Int 2009;30(10):945-949

24 Gadd RJ, Barwick TW, Paling E, Davies MB, Blundell CM. Assessment of a three-grade classification of complications in total ankle replacement. Foot Ankle Int 2014;35(5):434-437

25 Pyevich MT, Saltzman CL, Callaghan JJ, Alvine FG. Total ankle arthroplasty: a unique design. Two to twelve-year follow-up. J Bone Joint Surg Am 1998;80(10):1410-1420

26 Schuberth JM, Babu NS, Richey JM, Christensen JC. Gutter impingement after total ankle arthroplasty. Foot Ankle Int 2013;34(3): 329-337 